\title{
Promotion of cellular migration and apoptosis resistance by a mouse eye-specific phosphatidylethanolamine-binding protein
}

\author{
YUANYUAN ZHANG $^{1 *}$, XIAOJIAN WANG ${ }^{2 *}$, ZHENGHUA XIANG $^{3}$, HONGZHE LI $^{1}$, JIANMING QIU $^{1}$, \\ QIAOLING SUN ${ }^{1}$, TAO WAN ${ }^{4}, \mathrm{NAN} \mathrm{LI}^{4}$, XUETAO CAO ${ }^{1,2,4}$ and JIANLI WANG ${ }^{1}$ \\ ${ }^{1}$ Institute of Immunology, Zhejiang University, Hangzhou 310031; ${ }^{2}$ Institute of Immunology, \\ Tsinghua University, Beijing 100084; Institutes of ${ }^{3}$ Neuroscience, and ${ }^{4}$ Immunology, \\ Second Military Medical University, Shanghai 200433, P.R. China
}

Received July 21, 2006; Accepted September 18, 2006

\begin{abstract}
The phosphatidylethanolamine (PE)-binding protein (PEBP) family is an evolutionary conserved group of proteins found in various species with multiple functions. We identified human PE-binding protein 4 (hPEBP4), a novel member of the PEBP family, as an anti-apoptotic molecule. Here we describe the cloning and functional characterization of the mouse homolog of hPEBP4 (mouse PEBP4, mPEBP4). Fulllength cDNA of mPEBP4 contains 1048 bp with an open reading frame (ORF) of $729 \mathrm{bp}$, which is predicted to encode a 242-aa protein. mPEBP4 contains a PE-binding domain, in this case between amino acids 106 and 213. mPEBP4 localizes primarily to endoplasmic reticulum/Golgi apparatus. Interestingly, RT-PCR and in situ hybridization analyses indicate that $\mathrm{mPEBP} 4$ is specifically expressed in mouse eye tissue. We demonstrate that mPEBP4 promotes cellular migration and invasion by inhibiting ERK1/2 and JNK activation and up-regulating the expression of COX-2. In addition, mPEBP4 overexpression inhibits Epirubicin-induced cellular apoptosis. Considering that mPEBP4 is specifically expressed in retinal ganglion cells, whether mPEBP4 is an important molecule involved in visual function needs to be further investigated.
\end{abstract}

\section{Introduction}

Many cytosolic proteins share the ability to specifically bind phospholipids, such as phosphatidylethanolamine (PE).

Correspondence to: Dr Jianli Wang, Institute of Immunology, Zhejiang University, 353 Yan'an Road, Hangzhou 310031, P.R. China E-mail: jlwang@zju.edu.cn

Dr Xuetao Cao, Institute of Immunology, Second Military Medical University, 800 Xiangying Road, Shanghai 200433, P.R. China

E-mail: caoxt@public3.sta.net.cn

${ }^{*}$ Contributed equally

Key words: molecular cloning, phosphatidylethanolamine-binding protein, cell migration, apoptosis, eye
Over the past decade, various members of the PE-binding protein (PEBP) family have been identified. This family is an evolutionarily conserved group of 21- to $23-\mathrm{kDa}$ basic proteins found in various plant and animal organisms such as Arabidopsis (1-3), tomato (1-3), tobacco (1-3), Saccharomyces cerevisiae (4), Onchocerca volvulus (5), Caenorhabditis elegans (6), and Drosophila melanogaster (7), as well as in mammals (human, rat, mouse, and bovine) $(8,9)$. The members of the PEBP family may be multifunctional; they have been implicated in membrane biogenesis $(10,11)$, membrane fluidity and the formation of functional domains (12), the stimulation of acetylcholine secretion during neuronal development (13), and serine protease inhibition in neuronal tissue (14).

Human PE-binding protein 4 (hPEBP4), a novel member of the PEBP family, was isolated from the human bone marrow stromal cell cDNA library by large-scale random sequencing by us (15). We demonstrate that hPEBP4 is highly expressed in human breast cancer and acts as an antiapoptotic molecule. Once hPEBP4 is silenced, the breast cancer cells became more sensitive to TNF $\alpha$-induced cellular apoptosis, indicating that the hPEBP4 may be a candidate target for breast cancer therapeutics $(15,16)$. On the basis of previous studies of hPEBP4, we aimed to identify the mouse homolog of hPEBP4 (mouse PEBP4, mPEBP4) and characterize its functions. Here we report the cloning and functional characterization of mPEBP4. More interestingly, mPEBP4 has been found to be specifically expressed in mouse eye tissue. We demonstrate that overexpression of mPEBP4 promotes cellular migration and inhibits cellular apoptosis by inhibiting activation of ERK1/2, JNK and upregulating COX-2 expression.

\section{Materials and methods}

Reagents and cell culture. TNF $\alpha$ and TRAIL were obtained from PeproTech (Rocky Hill, NJ). COX-2 inhibitor NS398 was obtained from Calbiochem (San Diego, CA). All of the cells were grown in RPMI-1640 or DMEM supplemented with $10 \%$ (v/v) fetal calf serum, $4.5 \mathrm{~g} / \mathrm{l} \mathrm{D}$-glucose, nonessential amino acids (100 uM each), $100 \mathrm{units} / \mathrm{ml}$ penicillin, $100 \mu \mathrm{g} / \mathrm{ml}$ streptomycin, and $2 \mathrm{mM}$ glutamine at $37^{\circ} \mathrm{C}$ in a $5 \% \mathrm{CO}_{2}$ atmosphere. 
Table I. Sequences of specific primers for cellular migration-related molecules.

\begin{tabular}{ll}
\hline Gene & \multicolumn{1}{c}{ Primer sequence } \\
\hline MMP-3 sense & 5'- GTC TCA AGA TGA TAT AAA TG-3' \\
MMP-3 antisense & 5'- AAT TGA TTT CCT TTA AAA ATG A-3' \\
MMP-9 sense & 5'-CCC GGA CCA AGG ATA CAG-3' \\
MMP-9 antisense & 5'-CAG TAC CGA GAG AAA GCC-3' \\
COX-2 sense & 5'-GCT TCC ATT GCC AGA GCA GGC A-3' \\
COX-2 antisense & 5'-GAG CTC TGG ATC TGG AAC ACT G-3' \\
TGF-3 sense & 5'-CAC CAA CTA TTG CTT CAG C-3' \\
TGF- 3 antisense & 5'-GAT CAT GTT GGA CAG CTG-3' \\
CCR7 sense & 5'-CATCAGCATTGACCGCTACGT-3' \\
CCR7 antisense & 5'-GGTACGGATGATAATGAGGTAGCA-3' \\
CXCR4 sense & 5'-ATG TCC ATT CCT TTG CCT CTT TTG C-3' \\
CXCR4 antisense & 5'-TTA GCT GGA GTG AAA ACT TGA AGA C-3' \\
\hline
\end{tabular}

Cloning of full-length mPEBP4 cDNA. According to the sequence on GenBank (GenBank accession no. AK006964), we designed the primers of mPEBP4 and cloned the full length of mPEBP4 from mouse eye cDNA. The fragment of mPEBP4 was amplified by the forward primer (F) 5'-GC GAA TTC ATG ACA ATG AAG CTG GTC G-3' and reverse primer (R) 5'-G CAA GCT TGA TTT TTG ATT GAA TTG CT-3' and the PCR products were purified and cloned into pMD18-T (Takara) vector and then sequenced.

Plasmid construction and cell transfection. To construct an expression vector for mPEBP4, the full-length coding region was subcloned into the expression vector pcDNA-3.1/mycHis(-)B vector (Invitrogen), to give mPEBP4-B. cDNA encoding the mPEBP4 was also cloned into a C-terminal GFP fusion expression vector (mPEBP4-GFP). Cell transfection using Lipofectamine reagent (Invitrogen) was performed as instructed by the manufacturer. Stable cells expressing mPEBP4 were established by selection with G418 (600$800 \mu \mathrm{g} / \mathrm{ml}$ ) for three weeks and confirmed by RT-PCR.

RT-PCR analysis. RT-PCR was performed as described previously (17). For detection of mPEBP4 mRNA expression in various cells and mouse tissues, total RNA extraction and first strand cDNA synthesis was performed. Amplification of the mPEBP4 fragment with the primers 5'-CTG GTC GCA GCAGCA CTT TG-3' (sense) and 5'-TTG CCA CGG ATA CTC CCA CT-3' (antisense) was performed for 30 cycles (30 sec at $94^{\circ} \mathrm{C}, 30 \mathrm{sec}$ at $56^{\circ} \mathrm{C}, 30 \mathrm{sec}$ at $72^{\circ} \mathrm{C}$ ). For detection of the inducible expression pattern of mPEBP4, breast cancer 4T1 cells were treated with Epirubicin $(1 \mu \mathrm{g} / \mathrm{ml}), \mathrm{TNF}-\alpha$ $(50 \mathrm{ng} / \mathrm{ml})$, or TRAIL $(100 \mathrm{ng} / \mathrm{ml})$ for indicated times. The sequences of the specific primers for cellular migration-related molecules are shown in Table I (18-23).

Observation of mPEBP4 cytolocalization by fluorescence confocal microscopy. HEK 293 cells transiently transfected with mPEBP4-GFP, or GFP control vector, growing on glass cover slides, were placed in 6-well plates, and then incubated with $0.5 \mu \mathrm{M}$ BODIPY 558/568 BFA derivatives (Molecular Probes) for $15 \mathrm{~min}$ at room temperature in the dark. Samples were washed briefly in PBS and then fixed in $4 \%$ polyformaldehyde for $10 \mathrm{~min}$ at room temperature prior to observation by fluorescence confocal microscopy (LSM confocal microscope; Carl Zeiss, Inc. Atlanda, GA).

In situ hybridization. In situ hybridization was carried out as according to the Nonradioactive In Situ Hybridization Application Manual provided by Roche Diagnostics Corp. with some modifications (24). Before in situ hybridization, frozen sections of mouse eye tissue were thawed at $42^{\circ} \mathrm{C}$ for $20 \mathrm{~min}$ and fixed for $10 \mathrm{~min}$ in $4 \%$ paraformaldehyde. Sections were then immersed in $1 \mathrm{mg} / \mathrm{l}$ proteinase $\mathrm{K}$ for several minutes and postfixed in $4 \%$ paraformaldehyde for $10 \mathrm{~min}$. After washing in $1 \mathrm{X}$ PBS for $10 \mathrm{~min}$ at room temperature, the sections were treated with $0.25 \%$ acetic anhydride and $0.1 \mathrm{~mol} / \mathrm{l}$ triethanolamine for $10 \mathrm{~min}$. Then the sections were rinsed with 1X PBS and dehydrated with serially increasing concentrations of alcohol. After dehydration, the sections were incubated in prehybridization buffer for $2 \mathrm{~h}$ at $42^{\circ} \mathrm{C}$. Then, $1 \mathrm{~g} / \mathrm{l}$ of digoxigenin-labeled sense or antisense riboprobes was diluted in hybridization buffer and added to the treated specimens. The sense probes were used as the control. The sense and antisense riboprobes were hybridized at $60^{\circ} \mathrm{C}$ for $18-22 \mathrm{~h}$. After hybridization, all unbound cRNA probes were highly stringently washed. Subsequently, sections were blocked in blocking buffer for $30 \mathrm{~min}$ at room temperature, then incubated with an anti-digoxigenin antibody conjugated to alkaline phosphatase diluted 1:2000 in blocking buffer overnight at $4^{\circ} \mathrm{C}$. Sections were then stained with NBTPBCIP overnight at room temperature. Finally, sections were mounted, observed and photographed.

Assay for cellular migration and invasion. Migration and invasion assay was performed as described previously (25-28). Transwell polycarbonate membranes ( $8-\mu \mathrm{m}$ pore size; Corning Costar Co., Cambridge, MA) coated with type IV collagen $(25 \mu \mathrm{g} / \mathrm{ml}$ ) or Matrigel-coated invasion chambers (Biocoat 
Matrigel invasion chamber, $8-\mu \mathrm{m}$ pore size; Becton Dickinson Labware, Bedford, MA) were used for assay of migration and invasion respectively. The stable transfectants of 4T1 cells $\left(5 \times 10^{4}\right)$ suspended in RPMI-1640 medium were added to the upper chamber. Lower chambers contained $10 \%$ serum. After $24 \mathrm{~h}$, cells were scraped from upper filter surfaces, and the cells on the lower surfaces were stained and counted. Data was shown as \% of control.

Western blot analysis. A BCA protein assay reagent kit (Pierce, Rockford, USA) was used to measure protein concentration. Total cell lysates were prepared by lysis buffer (Cell Signaling). The lysates were fractionated by $10 \%$ SDS-PAGE gel and transferred onto a nitrocellulose membrane (Schleicher \& Schuell). The blots were probed with antibodies specific for phospho-ERK1/2, phospho-JNK, phospho-p38, phospho-Akt (Cell Signaling), and then incubated with appropriate HRP-coupled secondary antibody (New England Biolabs, Mississauga, CA). Relevant protein bands were visualized using LumiGLo reagent (Cell Signaling).

Assay for cellular apoptosis. Apoptosis assay was performed as described previously (15). The cells were washed, resuspended in staining buffer, and stained with PI and R123 (R-302; Molecular Probes), according to the manufacturer's instructions. Stained cells were analyzed by FACScalibur (Becton Dickinson).

Statistics. Data are reported as mean values \pm SEM and compared with Student's t-test. Values $<0.05$ were considered significant.

\section{Results}

Molecular cloning and identification of mPEBP4. Through homology analysis of the PEBP family and bioinformatics search, we found an unknown protein from Mus musculus (GenBank accession no. AK006964), which contains a PEbinding domain, in this case between amino acids 106-213. As shown in Fig. 1, the novel cDNA was predicted to contain an ORF of $729 \mathrm{bp}$, encoding a 242-aa protein with a calculated molecular mass of 26,907 Da and an isoelectric point of 5.69. Homology analysis revealed close similarity (59 64\%) to other known members of the PEBP family from bovine, canis, human, orangutan, mouse, Xenopus laevis and others. At the primary amino acid level, it shared the highest homology with hPEBP4 which is identified as an anti-apoptosis molecule by us ( $45 \%$ identity and $57 \%$ similarity).

According to the report by O'Bryan and colleagues (29), previously identified PEBPs can be grouped into three subfamilies. The majority of differences between these subfamilies are in the $\mathrm{N}$-terminal residues (those preceding the PE-binding domain), especially the first 10 amino acids. Members of the three subfamilies share similar overall features, including length (each being $\sim 190$ residues) and amino acid composition in functional domains. The unnamed mouse protein BAB24810 is more than 220 residues in length, and contains at least 2 insertions and 1 deletion in their protein sequence, when compared to proteins belonging to the three existing subfamilies. So, we named BAB24810 as mouse PEBP4. The
mPEBP4 cDNA corresponded to Unigene cluster Hs.73523, located on mouse chromosome 14D1.

The residues, which were previously demonstrated to be conserved among all members of the family (Pro119, Asp120, Pro122, His134 and Arg1719), were also found in mPEBP4. These residues are thought to be involved in determining the local structure of a biologically important ligand-binding site in PEBPs. The two main regions of high sequence conservation occurred between residues 113 and 139 and residues 163 and 175 (based on mPEBP4 numbering). Residues 118-120 with 113-123 and 122 forming an Asp-Pro-Asp-X-Pro motif are universally conserved in all family members. The 163-175 conserved region formed part of the second side of the binding pocket. These structural features suggested that mPEBP4 might possess PE binding properties similar to those seen in other PEBPs.

$m R N A$ expression pattern of $m P E B P 4$. The mRNA expression pattern of mPEBP4 in normal mouse tissues was examined by RT-PCR analysis. The data showed that mPEBP4 mRNA was specifically expressed in mouse eye tissue and, therefore, we cloned the full-length cDNA of mPEBP4 from mouse eye tissue. We did not detect its expression in any other tissues including bone marrow, uterus, prostate, smooth muscle, stomach, liver, brain, hippocampus, skeletal muscle, lung, spleen, skin, colon, small intestine, thymus, ovary, rectum, heart, esophagus, mammary gland, or kidney (Fig. 2A and B). We also examined the expression pattern of mEBP4 mRNA in a variety of tumor cells and freshly isolated cells including mouse dendritic cells, macrophage, CD4 ${ }^{+} \mathrm{T}$ cells, A20 (B cell lymphoma), RAW264.7 (monocyte/macrophage), J774 (monocyte/macrophage), L929 (fibroblast), B16F10 (melanoma), B16 (melanoma), Hepa (liver carcinoma), 3LL (lung carcinoma), 4T1 (breast carcinoma), CT26 (colon carcinoma), and found that mPEBP4 was only weakly expressed in NIH3T3 fibroblast cells (Fig. 2C).

By using in situ hybridization, we further proved that mPEBP4 mRNA was specifically expressed in mouse eye tissue. We first examined every section of mouse eye tissue including cornea, iris, lens and retina, and found that mPEBP4 mRNA was specifically expressed in retina tissue. Moreover, a strong mPEBP4 mRNA hybridization signal was specifically detected in the ganglion cell layer (Fig. 2D, I) of retina tissue, no signal was detected in other layers such as the bipolar layer (Fig. 2D, II), layer of rods and cones (Fig. 2D, III) and pigment epithelium layer (Fig. 2D, IV). As shown in Fig 2D (left), anti-sense probes of mPEBP4 hybridized to the ganglion cell layer of retina tissue. No signal was observed in controls using sense probes (Fig. 2D, right).

Because hPEBP4 was found to be highly expressed in breast cancer cells and tissues but mPEBP4 was not detectable in mouse breast cancer cells, we questioned whether mPEBP4 expression could be induced by some cytotoxic agents. Therefore, the inducible expression of mPEBP4 mRNA in mouse breast cancer 4T1 cells treated with cytotoxic agents was further examined. As shown in Fig. 2E, following Epirubicin treatment, mPEBP4 expression increased in the 4T1 cells, reaching a maximum at $24 \mathrm{~h}$ and then decreasing, whereas TNF $\alpha$ or TRAIL had no effect on mPEBP4 expression in 4T1 cells (data not shown). The data suggest that mPEBP4 might 


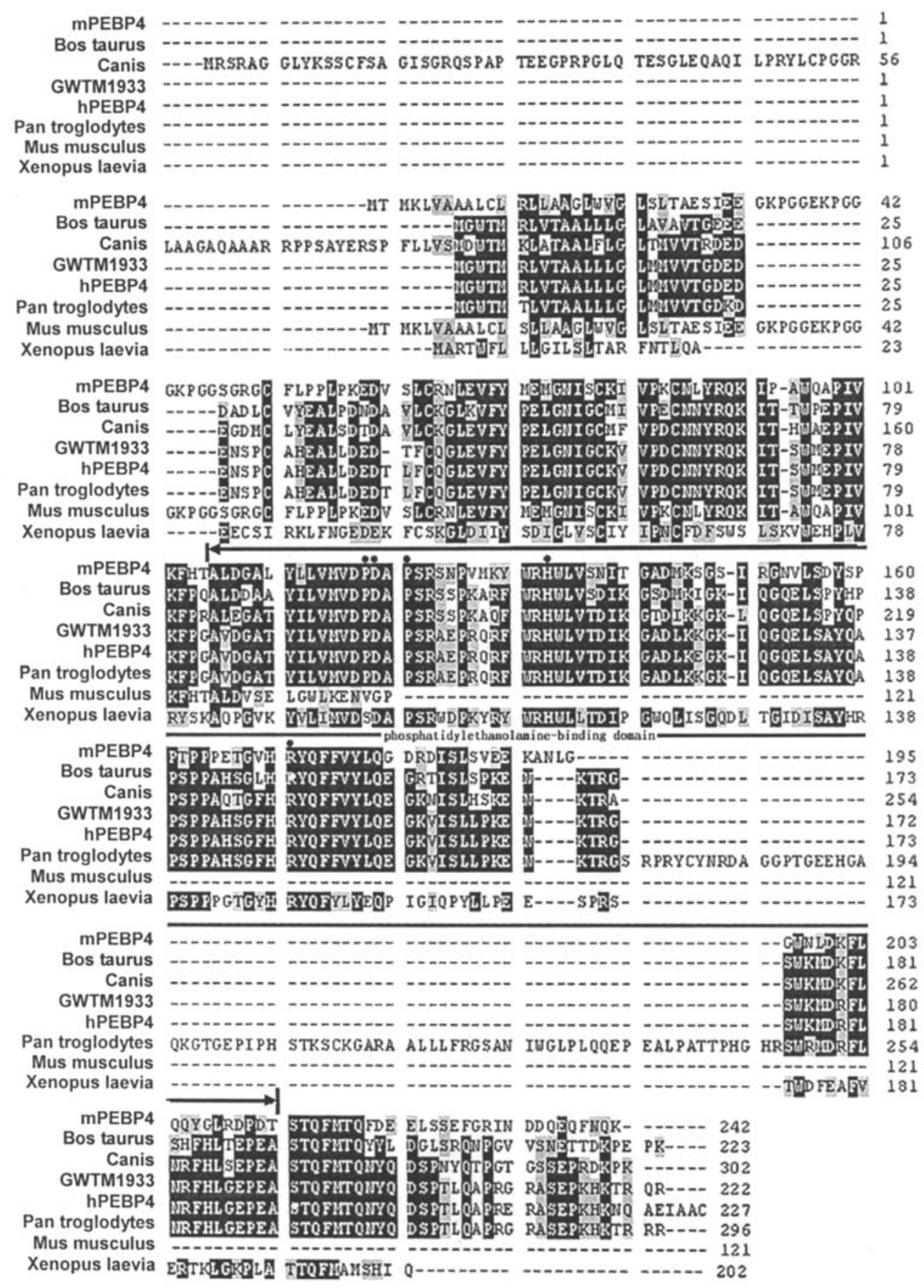

Figure 1. Multiple alignment of mPEBP4 with closely related PEBP family members. Alignment was performed with the GCG package and minimally adjusted manually. Identical residues are boxed in black, and similar residues are in gray. The phosphatidylethanolamine-binding domain is indicated by a bold arrow spanning residues 84-191 of mPEBP4. •, residues that are important for PE-binding. mPEBP4 sequence has been deposited in the GenBank database with the accession no. AK006964.

play roles in cellular apoptotic responses to extracellular stimuli such as Epirubicin.

Cytolocalization of $m P E B P 4$. The cytolocalization of GFPfused mPEBP4 protein in HEK 293 cells was examined by fluorescence confocal microscopy. Forty-eight hours after transfection with mPEBP4-GFP, GFP fluorescence displayed a cytoplasmic distribution in transfected cells, with a particularly strong signal in the perinuclear region. Further subcellular localization analysis was performed using specific probes for cell organelles including mitochondria, endoplasmic reticulum/ Golgi apparatus, and lysosomes. The majority of mPEBP4-
GFP green staining correlated with the red endoplasmic reticulum/Golgi apparatus signal (BFA Tracker), as displayed by yellow fluorescence in overlaid images (Fig. 2F), but did not correlate with red staining associated with mitochondria, or lysosomes (data not shown). Cells transfected only with GFP showed fluorescence in both cytoplasm and nucleus and no evidence of colocalization with endoplasmic reticulum/ Golgi apparatus. The results suggest that mPEBP4 localizes primarily to endoplasmic reticulum/Golgi apparatus.

mPEBP4 promotes the migration and invasion of breast cancer $4 T 1$ cells in vitro. Though mPEBP4 is specifically 




B
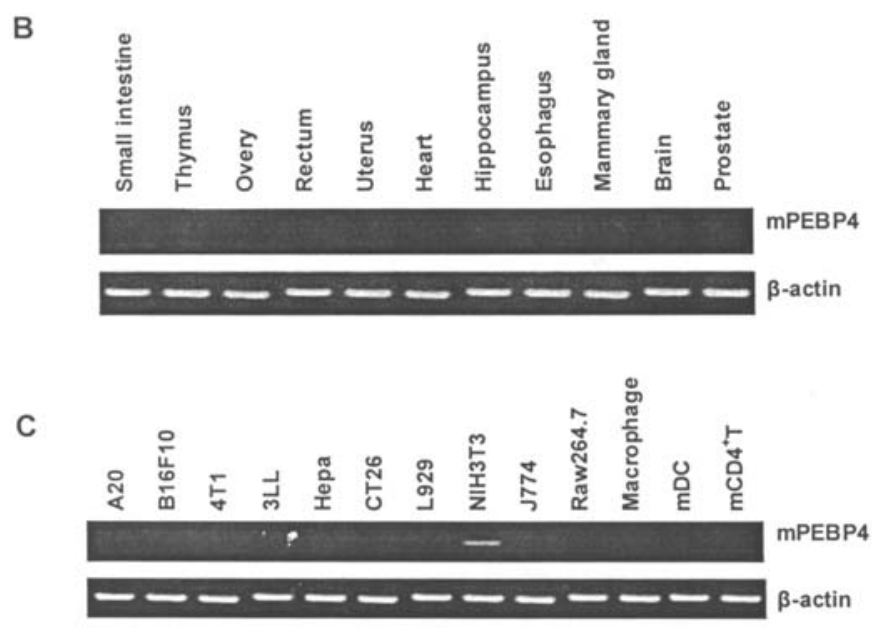

D

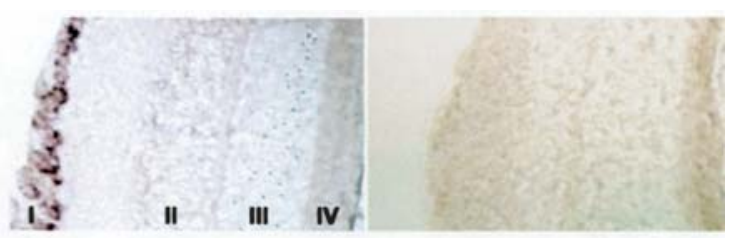

E

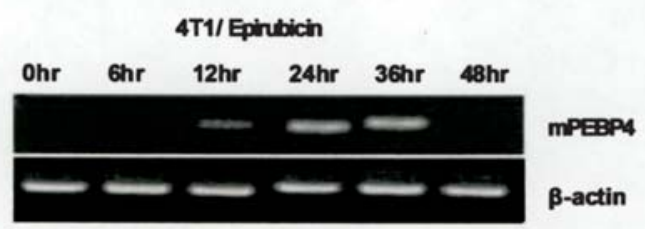

$\mathbf{F}$

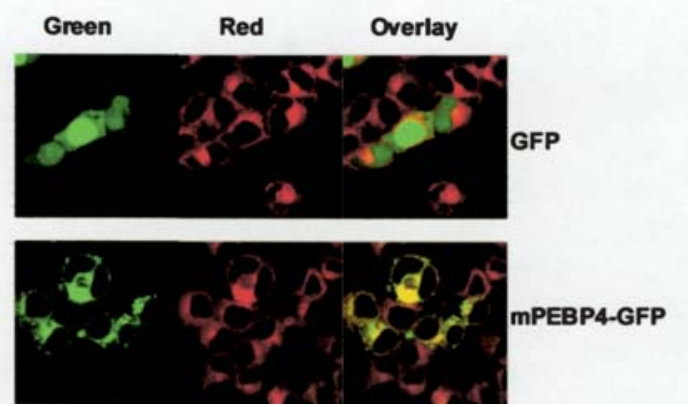

Figure 2. Expression pattern of mPEBP4 and subcellular localization of mPEBP4. RT-PCR analysis of mPEBP4 mRNA expression in mouse various tissues (A-B), and various cell lines (C) is shown. RT-PCR with mPEBP4 and mouse B-actin-specific primers was performed for 30 and 25 cycles, respectively. A 446-bp band of mPEBP4 was detected. All the tissues and cells were similarly positive for B-actin (lower panel). (D) Localization of mPEBP4 mRNA in the eyes of mouse by in situ hybridization. (a) Strong mPEBP4 mRNA hybridization signal was detected in the ganglionic layer of mouse eyes. I, ganglion cell layer; II, bipolar layer; III, layer of rods and cones cell; IV, pigment epithelium layer. (b) The negative control. No mPEBP4 mRNA hybridization signal was detected in the ganglion cell layer of mouse eyes. (E) Inducible expression pattern of mPEBP4 in mouse breast cancer 4T1 cells, which do not express mPEBP4 mRNA in normal state. Expression levels of mPEBP4 mRNA in 4T1 cells stimulated with $1 \mu \mathrm{g} / \mathrm{ml}$ Epirubicin were determined by RT-PCR at the indicated time-points. All the cells were similarly positive for B-actin (lower panel). (F) HEK 293 cells were transiently transfected with mPEBP4-GFP, or control GFP vector. The cells were fixed and fluorescence observed in overlay images $48 \mathrm{~h}$ after transfection.

expressed in mouse eye tissue, it is difficult to find an ideal mouse eye tissue-derived cellular model to explore the endogenous function of mPEBP4. Considering the inducible timedependent $\mathrm{mPEBP} 4$ expression pattern in mouse breast cancer 4T1 cells, 4T1 cells were selected here to investigate the function of mPEBP4. Because 4T1 cells have been proved to be an ideal tumor migration model $(30,31)$, we first investigated whether mPEBP4 could affect the migration and invasion of 4T1 cells. mPEBP4-B or control vector was transfected into 4T1 cells. RT-PCR confirmed the overexpression of mPEBP4 in 4T1 stable transfectants (Fig. 3A). The stable transfectants and parallel 4T1 cells were cultured in Transwell chambers for $24 \mathrm{~h}$. We found that the number of cells which migrated (Fig. 3B and D) and invaded (Fig. 3C and E) to the opposite sides of filters was much higher in mPEBP4-overexpressing 4T1 cells, indicating that mPEBP4 might have a facilitative effect on the migration and invasion of breast cancer 4T1 cells. A similar result was obtained from mPEBP4-overexpressed mouse fibroblast NIH3T3 cells (data not shown). The effect of mPEBP4 overexpression on 4T1 cell proliferation was simultaneously investigated, however, there was no statistically significant difference in proliferation rates between mPEBP4-B transfectants and mock transfectants or 4T1 cells (data not shown) suggesting that mPEBP4 could not enhance the growth of $4 \mathrm{~T} 1$ cells. These data demonstrate that $\mathrm{mPEBP} 4$ can promote cellular migration and invasion of 4T1 cells.

mPEBP4 overexpression inhibits serum-induced activation of ERK1/2 and JNK and up-regulates the expression of COX-2. It's well known that MAPK and PI3K/Akt pathways are important signaling pathways which account for cell migration $(32,33)$. So, we examined whether these signal pathways were involved in the promoting effect of mPEBP4 on the migration and invasion of 4T1 cells. As shown in Fig. 4A, serum-induced phosphorylation of ERK1/2 and JNK in 4T1 cells was inhibited by mPEBP4 overexpression, whereas activation of p38 and AKT was not influenced (data not shown).

Because some molecules, such as COX-2, MMP-3/9, TGF- 3 , CCR7 and CXCR4 have positive effects on cellular migration (34-37), we also investigated weather mPEBP4 could affect the expression of these molecules. RT-PCR and Western Blot analysis showed that only COX-2 expression was up-regulated in mPEBP4-overexpressing 4T1 cells (Fig. 4B and C). To determine whether the positive effect of mPEBP4 on cellular migration was linked to COX-2 expression, 4T1 cells stably transfected with mPEBP4-B, or 
A

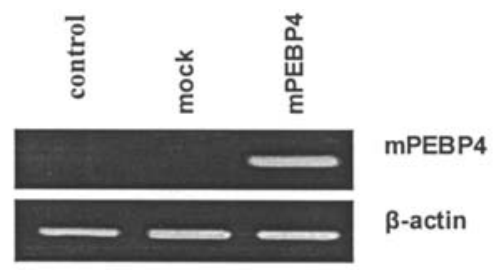

B

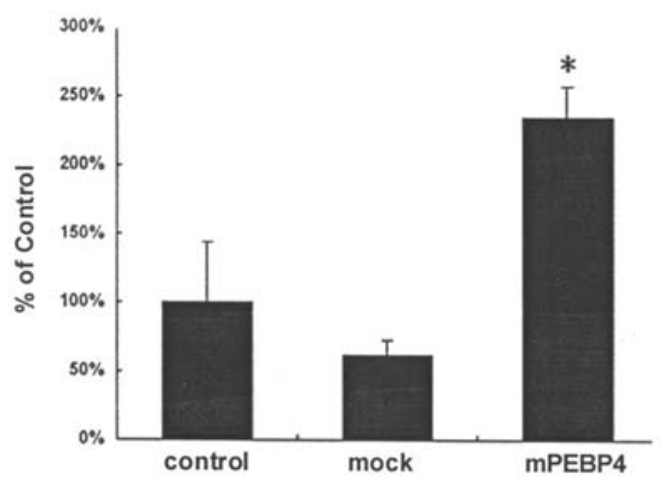

C



D

control

mock

MPEBP4

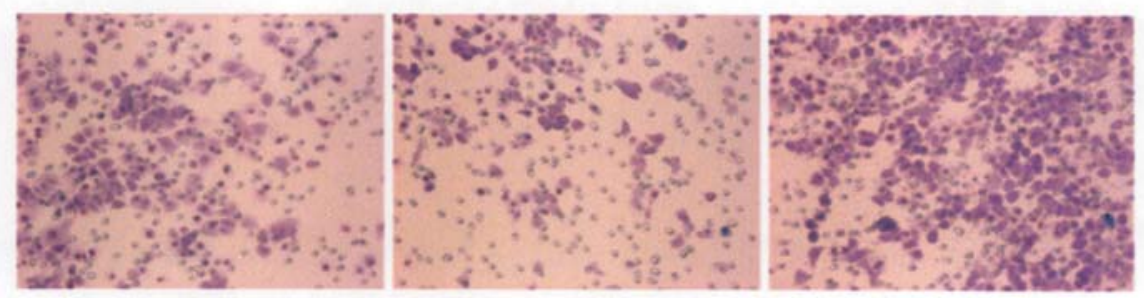

E


Figure 3. Effects of mPEBP4 overexpression on migration and invasion of breast cancer $4 \mathrm{~T} 1$ cells $i n$ vitro. Assays for migration and invasion of the cells were carried out by using Transwell chambers consisting of $8.0-\mu \mathrm{m}$ pore size filters, but only the filters used for the invasion assay were coated with Matrigel on the upper surface. (A) Stable expression of mPEBP4 in 4T1 cells, as confirmed by RT-PCR using mPEBP4-specific primers. Quantitative analysis of the effects of mPEBP4 overexpression on 4T1 cellular migration (B) and invasion (C). The number of cells which migrated and invaded to the opposite sides of filters in parallel 4T1 cells was given the value 100\%, such that migration and invasion of tansfectants were represented as a fraction of control. (D) Cells migrating through the pore of membranes were stained with Giemsa. The images were shown by fluorescence microscope. (E) The images of the invasion assay. Data of one representative experiment from the three independent experiments was shown and expressed as mean \pm SD from triplicate wells. Significant differences from control and mock are shown $\left({ }^{*} \mathrm{P}<0.05\right)$. 
A

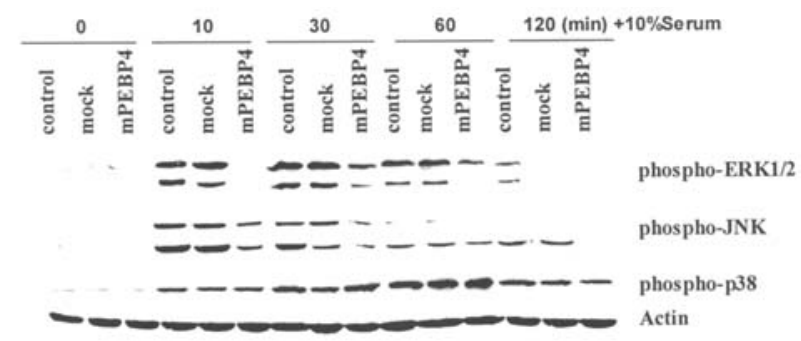

B

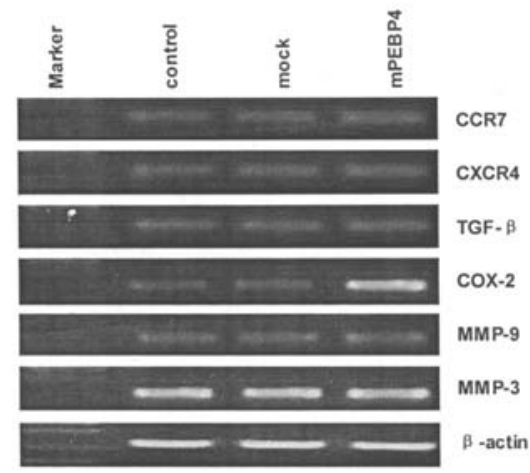

C
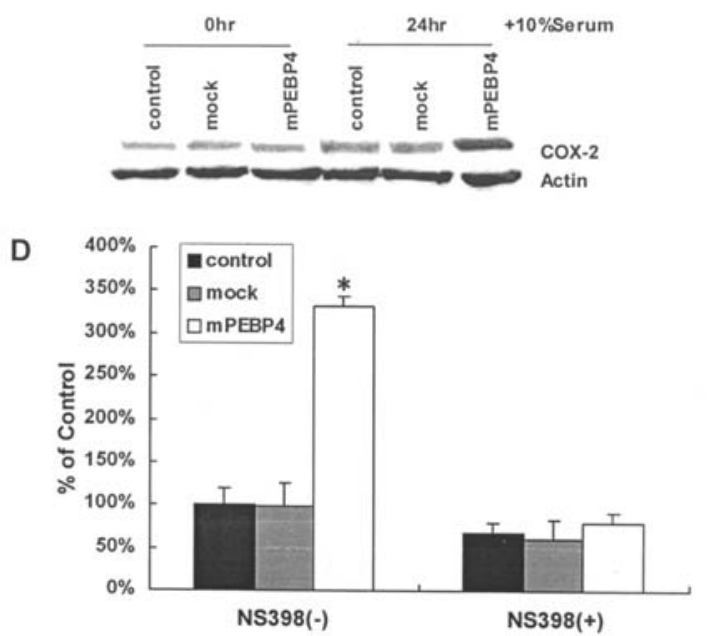

Figure 4. Overexpression of mPEBP4 inhibits serum-induced activation of ERK1/2 and JNK and up-regulates the expression of COX-2. (A) 4T1 cells stably transfected with mPEBP4-B, or mock and parallel 4T1 cells were serum-starved for $24 \mathrm{~h}$ and then treated with $10 \%$ serum for various periods of time. Equivalent protein loadings of each lysate were immunoblotted with antibodies recognizing the phosphorylated, active forms of pp42/pp44 ERK, p-JNK or p-p38. (B) mPEBP4-B, mock stably transfected 4T1 cells, or parallel 4T1 cells were serum-starved for $24 \mathrm{~h}$ and then treated with $10 \%$ serum for $24 \mathrm{~h}$. The cells were harvested for detection of migration and invasion-related molecules by RT-PCR. All the cells were similarly positive for $B$-actin (lowest panel). (C) Western blot analysis for the expression of COX-2. (D) mPEBP4 promotes the migration of 4T1 cells through upregulating the expression of COX-2. The stable transfectants of $4 \mathrm{~T} 1$ cells were grown in the upper chamber of a Transwell containing NS398 $(50 \mu \mathrm{M})$ or not for $24 \mathrm{~h}$, then migration assay was followed.

mock and parallel $4 \mathrm{~T} 1$ cells were treated with or without a selective COX-2 inhibitor (38), NS398 $(50 \mu \mathrm{M})$ for $24 \mathrm{~h}$. We found that the migratory capacity of $4 \mathrm{~T} 1$ cells promoted by mPEBP4 overexpression was significantly reduced by NS398 (Fig. 4D). A similar result was obtained in invasion assay (data not shown). These results suggested that mPEBP4 might promote the migration and invasion of mouse breast cancer $4 \mathrm{~T} 1$ cells by up-regulating the expression of COX-2.

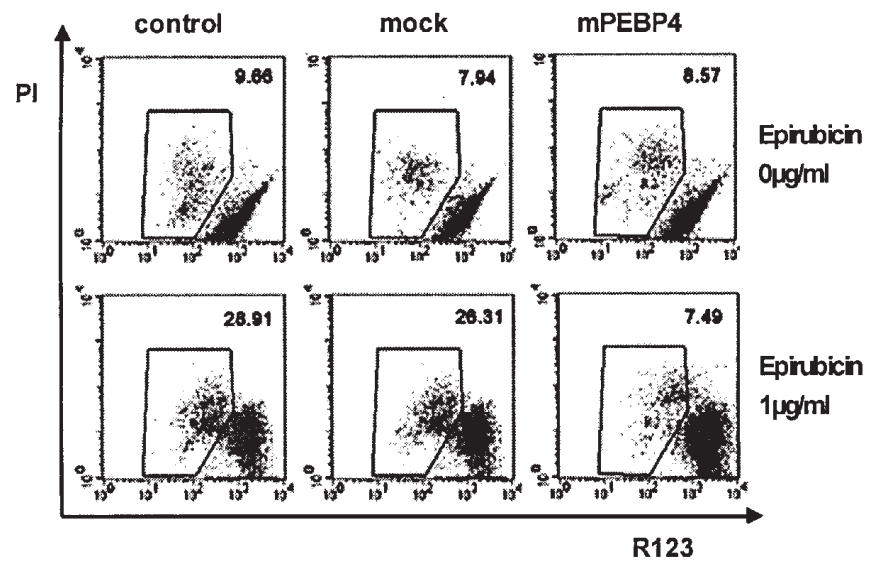

Figure 5. mPEBP4 overexpression inhibits Epirubicin-induced apoptosis. The stable transfectants of $4 \mathrm{~T} 1$ cells were treated with $1 \mathrm{ug} / \mathrm{ml}$ Epirubicin for $24 \mathrm{~h}$. Cells labeled by green fluorescent cationic dye (Rhodamine 123, R302) and PI. The percentages of the gated cells represent apoptotic cells.

MPEBP4 overexpression inhibits Epirubicin-induced apoptosis in breast cancer $4 T 1$ cells. Our previous study showed that human PEBP4 functions as an anti-apoptosis molecule (15), so the role of mPEBP4 in the process of cellular apoptosis was explored. The stable transfectants were treated with an indicated concentration of Epirubicin which had been identified as a kind of apoptosis-inducing agent in treating breast carcinoma (39). Loss of mitochondrial inner transmembrane potential is associated with the early stages of apoptosis and may be one of the central features of the process (40). The green fluorescent cationic dye Rhodamine 123 (R-302) binds selectively to the inner mitochondrial membrane and accumulates in the charged membrane compartments of living cells; apoptotic cells exhibit a loss of R123 binding. mPEBP4-B transfectants did not differ in their baseline level of apoptosis, when compared with mock transfectants or 4T1 cells, but did exhibit lower levels of apoptosis following Epirubicin treatment (Fig. 5). However, mPEBP4 had no effect on TNF $\alpha$ - or TRAIL-induced apoptosis in 4T1 cells (data not shown). A similar result was obtained from mPEBP4-overexpressed mouse fibroblast NIH3T3 cells (data not shown). Considering the inducible mPEBP4 expression pattern by Epirubicin, mPEBP4 might play roles in cellular apoptotic responses to Epirubicin treatment and in self-protection from Epirubicin-induced apoptosis in breast cancer cells. How mPEBP4 exerts its anti-apoptotic function needs to be investigated in the future.

\section{Discussion}

The PEBP family is a highly conserved group of proteins with homologues in a wide variety of organisms. Our previous studies showed that hPEBP4 functions as an anti-apoptotic molecule and might be a candidate target for breast cancer therapeutics $(15,16)$. In this study, we cloned and functionally identified mPEBP4, the mouse homolog of hPEBP4. To our interest, we found mPEBP4 was specifically expressed in eye tissue, however, until now, we had no ideal models for the investigation of mPEBP4 functions in mouse eye development and pathophysiological roles of MPEBP4 in the eye diseases. 
On the basis of Epirubicin-inducible mPEBP4 expression, we selected mouse breast cancer cells $4 \mathrm{~T} 1$ as the cellular model to investigate the function of mPEBP4. Our previous studies showed that hPEBP4 has a negative effect on TNF $\alpha$ or TRAILinduced apoptosis (15), here we found that mPEBP4 overexpression in breast cancer 4T1 cells conferred resistance to Epirubicin-induced apoptosis, but had no effect on $\mathrm{TNF} \alpha$ or TRAIL-induced apoptosis. Together with the fact that mPEBP4 mRNA expression was induced in 4T1 cells treated with Epirubicin but not TNF $\alpha$ or TRAIL, mPEBP4 might play roles in cellular apoptotic responses to cytotoxic agent Epirubicin and in self-protection from Epirubicin-induced apoptosis.

The contribution of MAPK activation to cellular migration depends on both cell type and stimulus. JNK has recently been shown to play an important role in the negative regulation of breast cancer cell migration (32). We found that mPEBP4 overexpression in mouse breast cancer 4T1 cells inhibited $10 \%$ serum-induced ERK, and JNK activation, but promoted cellular migration and invasion. However, mPEBP4 had no effect on p38, AKT, or NF-кB activation (data not shown). We also explored the effect of mPEBP4 overexpression on migrationrelated molecule expression, and found that only COX-2 expression was up-regulated in mPEBP4-overexpressing 4T1 cells. Furthermore, the selective COX-2 inhibitor NS398 inhibited the cellular migration and invasion promoted by mPEBP4 overexpression. Therefore, mPEBP4-mediated suppression of ERK and JNK activation and up-regulation of COX-2 expression appear to be partially responsible for the observed promotion of cellular migration and invasion by mPEBP4.

Most interestingly, in situ hybridization showed that mPEBP4 was specifically expressed in retinal ganglion cells which are the first or among the first cells born in the retina in all the studied vertebrates and play an important role in visual function (41). Recent study demonstrated that apoptosis of the retinal ganglion cell is characteristic of glaucoma. Many scientists are trying to find neuroprotective strategies to protect ganglion cells from apoptosis to battle against blinding diseases. Considering its anti-apoptosis effect, mPEBP4 may be an important molecule involved in visual function, and further experiments need to be conducted to confirm this prediction.

In conclusion, we have cloned and functionally characterized a mouse eye-specific protein mPEBP4, a novel member of the PEBP family, which shows similar structural features to hPEBP4. We demonstrate that mPEBP4 promotes cellular migration and invasion by inhibiting ERK1/2 and JNK activation and up-regulating the expression of COX-2. In addition, mPEBP4 overexpression inhibits Epirubicininduced cellular apoptosis. Considering that mPEBP4 is specifically expressed in retinal ganglion cells, whether mPEBP4 is an important molecule involved in visual function needs to be further investigated.

\section{Acknowledgements}

We sincerely thank Mrs. Mei Jin and Yan Li for their excellent technical assistance. This work was supported by grants from the National Natural Science Foundation of China (30121002), Tsinghua-Yue-Yuen Medical Sciences Fund (202400.005-02),
Shanghai Science Foundation, and the National Key Basic Research Program of China (2001CB510002).

\section{References}

1. Ohshima S, Murata M, Sakamoto W, Ogura Y and Motoyoshi F: Cloning and molecular analysis of the Arabidopsis gene Terminal Flower 1. Mol Gen Genet 254: 186-194, 1997.

2. Pnueli L, Carmel-Goren L, Hareven D, et al: The SELFPRUNING gene of tomato regulates vegetative to reproductive switching of sympodial meristems and is the ortholog of CEN and TFL1. Development 125: 1979-1989, 1998.

3. Amaya I, Ratcliffe OJ and Bradley DJ: Expression of CENTRORADIALIS (CEN) and CEN-like genes in tobacco reveals a conserved mechanism controlling phase change in diverse species. Plant Cell 11: 1405-1418, 1999.

4. Robinson LC and Tatchell K: TFS1: a suppressor of cdc 25 mutations in Saccharomyces cerevisiae. Mol Gen Genet 230: 241-250, 1991.

5. Erttmann KD and Gallin MY: Onchocerca volvulus: Identification of cDNAs encoding a putative phosphatidylethanolaminebinding protein and a putative partially processed mRNA precursor. Gene 174: 203-207, 1996.

6. Wilson R, Ainscough R, Anderson K, et al: $2.2 \mathrm{Mb}$ of contiguous nucleotide sequence from chromosome III of C. elegans. Nature 368: 32-38, 1994.

7. Adams MD, Celniker SE, Holt RA, et al: The genome sequence of Drosophila melanogaster. Science 287: 2185-2195, 2000.

8. Seddiqi N, Segretain D, Bucquoy S, et al: Characterization and localization of the rat, mouse and human testicular phosphatidylethanolamine binding protein. Experientia 52: 101-110, 1996.

9. Bernier I and Jolles P: Purification and characterization of a basic $23 \mathrm{kDa}$ cytosolic protein from bovine brain. Biophys Acta 790: 174-181, 1984

10. Moore C, Perry ACF, Love S and Hall L: Sequence analysis and immunolocalisation of phosphatidylethanolamine binding protein (PBP) in human brain tissue. Mol Brain Res 37: 74-78, 1996.

11. Frayne J, McMillen A, Love S and Hall L: Expression of phosphatidylethanolamine-binding protein in the male reproductive tract: immunolocalisation and expression in prepubertal and adult rat testes and epididymides. Mol Reprod Dev 49: 454-460, 1998 .

12. Tohdoh N, Tojo S, Agui H and Ojika K: Sequence homology of rat and human HCNP precursor proteins, bovine phosphatidylethanolamine-binding protein and rat $23-\mathrm{kDa}$ protein associated with the opioid-binding protein. Mol Brain Res 30: 381-384, 1995.

13. Oijka K, Mitake S, Tohdoh N, et al: Hippocampal cholinergic neurostimulating peptides (HCNP). Prog Neurobiol 160: 37-83, 2000 .

14. Hengst U, Albrecht H, Hess D and Monard D: The phosphatidylethanolamine-binding protein is the prototype of a novel family of serine protease inhibitors. J Biol Chem 276: 535-540, 2001.

15. Wang X, Li N, Liu B, et al: A novel human phosphatidylethanolamine-binding protein resists tumor necrosis factor alpha-induced apoptosis by inhibiting mitogen-activated protein kinase pathway activation and phosphatidylethanolamine externalization. J Biol Chem 279: 45855-45864, 2004.

16. Wang X, Li N, Li H, et al: Silencing of human phosphatidylethanolamine-binding protein 4 sensitizes breast cancer cells to tumor necrosis factor-alpha-induced apoptosis and cell growth arrest. Clin Cancer Res 11: 7545-7553, 2005.

17. Chen T, Guo J, Yang M, et al: Cyclosporin A impairs dendritic cell migration by regulating chemokine receptor expression and inhibiting cyclooxygenase-2 expression. Blood 103: 413-421, 2004.

18. Haupt LM, Thompson EW, Trezise AE, Irving RE, Irving MG and Griffiths LR: In vitro and in vivo MMP gene expression localisation by In Situ-RT-PCR in cell culture and paraffin embedded human breast cancer cell line xenografts. BMC Cancer 6: 1471-1481, 2006.

19. Moon HI and Chung JH: Meso-dihydroguaiaretic acid from Machilus thunbergii SIEB et ZUCC., and its effects on the expression of matrix metalloproteinase-2, 9 caused by ultraviolet irradiated cultured human keratinocyte cells (HaCaT). Biol Pharm Bull 28: 2176-2179, 2005. 
20. Johnson MC, Torres M, Alves A, et al: Augmented cell survival in eutopic endometrium from women with endometriosis: expression of c-myc, TGF-beta1 and bax genes. Reprod Biol Endocrinol 3: 7827-7835, 2005

21. Zhou H, Ivanov V, Gillespie NJ, et al: Mechanism of radiationinduced bystander effect: role of the cyclooxygenase-2 signaling pathway. Proc Natl Acad Sci USA 102: 14641-14646, 2005.

22. Kang H, Watkins G, Douglas JA, Mansel RE and Jiang WG: The elevated level of CXCR4 is correlated with nodal metastasis of human breast cancer. Breast 14: 360-367, 2005.

23. Fainaru O, Shseyov D, Hantisteanu S and Groner Y: Accelerated chemokine receptor 7-mediated dendritic cell migration in Runx3 knockout mice and the spontaneous development of asthma-like disease. Proc Natl Acad Sci USA 102: 10598-10603, 2005.

24. Tanaka K, Tamure H, Tanaka H, et al: Spermatogoniadependent expression of testicular genes in mice. Dev Biol 246: 466-479, 2002.

25. Muraoka RS, Dumont N, Ritter CA, et al: Blockade of TGFbeta inhibits mammary tumor cell viability, migration, and metastases. J Clin Invest 109: 1551-1559, 2002.

26. Bakin AV, Tomlinson AK, Bhowmick NA, Moses HL and Arteaga CL: Phosphatidylinositol 3-kinase function is required for transforming growth factor beta-mediated epithelial to mesenchymal transition and cell migration. J Biol Chem 275: 36803-36810, 2000.

27. Smith MC, Luker KE, Garbow JR, et al: CXCR4 regulates growth of both primary and metastatic breast cancer. Cancer Res 64: 8604-8612, 2004.

28. Fong S, Itahana Y, Sumida T, et al: Id-1 as a molecular target in therapy for breast cancer cell invasion and metastasis. Proc Natl Acad Sci USA 100: 13543-13548, 2003.

29. Hickox DM, Gibbs G, Morrison JR, et al: Identification of a novel testis-specific member of the phosphatidylethanolamine binding protein family, pebp-2. Biol Reprod 67: 917-927, 2002.

30. Hu L, Lee M, Campbell W, Perez-Soler R and Karpatkin S: Role of endogenous thrombin in tumor implantation, seeding, and spontaneous metastasis. Blood 104: 2746-2751, 2004.
31. Monzavi-Karbassi B, Whitehead TL, Jousheghany F, et al: Deficiency in surface expression of E-selectin ligand promotes lung colonization in a mouse model of breast cancer. Int $\mathrm{J}$ Cancer 117: 398-408, 2005.

32. Huang TJ, Price SA, Chilton L, et al: Insulin prevents depolarization of the mitochondrial inner membrane in sensory neurons of type 1 diabetic rats in the presence of sustained hyperglycemia. Diabetes 52: 2129-2136, 2003.

33. Fukaya Y, Ishiguro N, Senga T, et al: A role for PI3K-Akt signaling in pulmonary metastatic nodule formation of the osteosarcoma cell line, LM8. Oncol Rep 14: 847-852, 2005.

34. Sun W, Sun Y, Fang R, et al: Expression of cyclooxygenase-2 and matrix metalloproteinase-9 in gastric carcinoma and its correlation with angiogenesis. Jap J Clin Oncol 35: 707-713, 2005.

35. Yu Q and Stamenkovic I: Transforming growth factor-beta facilitates breast carcinoma metastasis by promoting tumor cell survival. Clin Exp Metastasis 21: 235-242, 2004.

37. Cabioglu N, Yazici MS, Arun B, et al: CCR7 and CXCR4 as novel biomarkers predicting axillary lymph node metastasis in T1 breast cancer. Clin Cancer Res 11: 5686-5693, 2005.

38. Chu J, Lloyd FL, Trifan OC, Knapp B and Rizzo MT: Potential involvement of the cyclooxygenase-2 pathway in the regulation of tumor-associated angiogenesis and growth in pancreatic cancer. Mol Cancer Ther 2: 1-7, 2003.

39. Small GW, Somasundaram S, Moore DT, Shi YY and Orlowshi RZ: Repression of mitogen-activated protein kinase (MAPK) phosphatase-1 by anthracyclines contributes to their antiapoptotic activation of p44/42-MAPK. J Pharmacol Exp Ther 307: 861-869, 2003.

40. Matsuyoshi S, Shimada K, Nakamura M, Ishida E and Konishi N: FADD phosphorylation is critical for cell cycle regulation in breast cancer cells. Br J Cancer 94: 532-539, 2006.

41. Huang W, Fileta JB, Dobberfuhl A, et al: Calcineurin cleavage is triggered by elevated intraocular pressure, and calcineurin inhibition blocks retinal ganglion cell death in experimental glaucoma. Proc Natl Acad Sci USA 102: 12242-12247, 2005. 